\title{
Better Foreground Segmentation for 3D Face Reconstruction Using Graph Cuts
}

\author{
Anjin Park, Kwangjin Hong, and Keechul Jung* \\ School of Digital Media, College of Information Science, Soongsil University \\ 156-743, Seoul, S. Korea \\ \{anjin, hongmsz, kcjung\} @ssu.ac.kr
}

\begin{abstract}
Research on image-based 3D reconstruction has recently shown a lot of good results, but it assumes precise target objects are already segmented from each input image. Traditionally, background subtraction was used to segment the target objects, but it can yield serious problems, such as noises and holes. To precisely segment the target objects, graph cuts have recently been used. Graph cuts showed good results in many engineering problems, as they can globally minimize energy functions composed of data terms and smooth terms, but it is difficult to automatically obtain prior information necessary for data terms. Depth information generated by stereo vision was used as prior information, which shows good results in their experiments, but it is difficult to calculate depth information for 3D face reconstruction, as the most of faces have homogeneous regions. In this paper, we propose better foreground segmentation method for $3 \mathrm{D}$ face reconstruction using graph cuts. The foreground objects are approximately segmented from each background image using background subtraction to assist to estimate data terms of energy functions, and noises and shadows are removed from the segmented objects to reduce errors of prior information. Removing the noises and shadows should cause to lose detail in the foreground silhouette, but smooth terms that assign high costs if neighboring pixels are not similar can fill out the lost silhouette. Consequently, the proposed method can segment more precise target objects by globally minimizing the energy function composed of smooth terms and approximately estimated data terms using graph cuts.
\end{abstract}

Keywords: Foreground Segmentation, Graph Cuts, Shadow Elimination, 3D Face Reconstruction.

\section{Introduction}

Since the world that we live is $3 \mathrm{D}$ space, we can feel the reality and get more valuable information from 3D objects. For this reason, research on 3D reconstruction for interest regions, such as various objects, human bodies, and faces, has recently attracted a lot of attention in the fields of computer vision and graphics. Specially, image-based $3 \mathrm{D}$ reconstruction that reconstructs $3 \mathrm{D}$ objects from images captured by

\footnotetext{
${ }^{*}$ Corresponding author.
} 
multi-cameras has shown good results with the rapid growth of computer performance and stable reconstruction algorithm[1].

The image-based 3D reconstruction has shown good results, but it assumes precise target objects, such as face regions for 3D face reconstruction, are already segmented from each input image, and traditionally background subtraction was used to segment objects. The background subtraction first builds a model of the static background, either off-line or updated dynamically, and then compares new input image with the background model on a per-pixel basis[2]. However, they tended to produce unstable and error-prone results, including false foreground blobs and holes in segmented foregrounds caused by camera noises or low contrast with background areas[3].

For better results, researchers have used graph cuts for foreground segmentation[410]. Graph cuts can globally minimize energy functions composed of data terms and smooth terms, and they shows high precision rates if prior information are given for data terms of energy functions. Li et al.[4], Boykov and Funka-Lea[5], and Rother et al.[6] proposed interactive image segmentation tools using graph cuts. In tools proposed by Li et al.[4] and Boykov and Funka-Lea[5], a user marks a few lines on the images by dragging the mouse cursor while holding a button, e.g. the left button indicates the foreground and the right button indicates the background. Rother et al.[6] proposed the interactive tool that the user loosely drags a rectangle around an objects to merely touch foreground in a few locations. Above mentioned methods shows high precision rates by segmenting foreground using graph cuts, but they can not automatically segment the foreground, which restricts the scope of applicability, as users manually gave prior information by lines[4,5] or rectangles[6].

Recently, depth information generated by stereo vision techniques was used as the prior information for data terms[7,8], based on an assumption that objects within specific scopes of depth are target objects. They showed good results in their experiments, but it is difficult to calculate accurate depth information in $3 \mathrm{D}$ face reconstruction, as the most of faces have homogeneous colors and textures, which means accurate prior information can not be obtained. As another approaches to foreground segmentation using graph cuts, Howe and Deschamps[10] used the difference between the background image and the input image as prior information for the background, and used given parameters for the foreground. Therefore, this approach was very sensitive to camera noises, and made many holes within segmented foreground owing to inaccurate prior information for the foreground. Sun et al.[11] used Gaussian mixture models(GMM) for prior information of the background, which is robust to the noises, but used constant values as prior information of the foreground based on an assumption that is uniform distribution in appearance of foreground objects, which restricts applicability of the proposed method.

This paper uses Volume Intersection[1] and Object Carving[9] that is the most popular method instead of stereo vision techniques. Volume Intersection tentatively reconstructs $3 \mathrm{D}$ face using silhouette of the face, and thus it needs accurate silhouette information. Object Carving is used for definitive 3D face reconstruction, and needs accurate foreground objects without noises and holes. Accordingly, this paper proposes better foreground segmentation method for 3D face reconstruction using graph cuts. To improve the precision rates for foreground segmentation, more 
accurate priori information for foreground objects is needed instead of given parameters. For this, the foreground objects are approximately segmented from each background image using background subtraction, and noises and shadow regions are removed from the segmented objects to reduce the errors of prior information for foreground objects. Removing the noises and shadows should cause to lose legitimate detail in the foreground silhouette, but smooth terms that assign high costs if neighboring pixels are not similar can fill out the lost silhouette. Consequently, the proposed method estimates more accurate prior information for foreground objects, and fills out the lost foreground objects based on smooth terms. Moreover, the graph cuts can segment more precise foreground objects by globally minimizing the energy function composed of smooth terms and data terms

The remainder of this paper is organized as follows. The proposed foreground segmentation method using graph cuts are described in section 2. Some experimental results and 3D face reconstruction using Volume Interaction and Object Carving are then presented in section 3 , and final conclusions are given in section 4 .

\section{Foreground Segmentation}

This paper uses graph cuts to improve the accuracy of foreground segmentation. Generally, when graph cuts are used for computer vision problems, two problems are encountered: how to express the energy function, and how to automatically obtain prior information for data terms. Section 2.1 describes how to express the energy function for foreground segmentation, and section 2.2 describes how to obtain prior information for the foreground and background.

\subsection{Energy Function for Foreground Segmentation}

This paper considers foreground segmentation as a labeling problem. The labeling problem is to assign a label from a set of labels, $\{$ ' $f g$ ' and ' $b g$ ' $\}$ where $f g$ means the foreground and $b g$ means the background, to each pixel in an input image $P$, and the labeling is denoted by $\mathbf{F}=\left\{f_{1}, f_{2}, \ldots, f_{D n}\right\}$, where $D n$ is the number of pixels and each element of $\mathbf{F}$ can be one of the label set. To assign a specific label to each node, the foreground segmentation problem is first expressed as an energy function(Eq. 1).

$$
E(\mathbf{F})=\sum_{p \in P} D_{p}\left(f_{p}\right)+\lambda \sum_{\{p, q\} \in N} V_{p, q}\left(f_{p}, f_{q}\right)
$$

Here, $D_{p}\left(f_{p}\right)$ is a data term that reflects how each pixel fits into the prior data given for each label. In other words, $D_{p}\left(f_{p}\right)$ has low costs if a pixel $p$ is similar to a label $f_{p}$, and has high costs if a pixel $p$ is not similar to a label $f_{p} \cdot V_{p, q}\left(f_{p}, f_{q}\right)$ is a smooth term that reflects discontinuities between neighboring pixel $p$ and $q$, and has high costs if two neighboring pixels are similar. $\lambda$ specifies the relative importance of two energy terms, and $N$ is all pairs of neighboring elements in $P$, called $n$-links(neighborhood links).

To minimize the energy function via graph cuts, a graph $G=\langle v, \varepsilon\rangle$ is first created with nodes corresponding to the pixels. Two distinguished nodes, $\operatorname{source}(S)$ and 
$\operatorname{sink}(T)$ called terminals, are also needed to represent two labels, plus each node has two additional edges, called t-links(terminal links) $\{p, S\}$ and $\{p, T\}$. Therefore, $v=P \cup\{S, T\}$ and $\varepsilon=N \underset{p \in P}{\cup}\{\{p, S\},\{p, T\}\}$. The weights of the graph are set for both the $t$-links and $n$-links, where t-links connecting each terminal and each node are correspond to the data that indicates the label-preferences of each pixel and n-links connecting between neighboring nodes are correspond to the smooth terms that indicates discontinuities between neighboring nodes. The graph $G$ is then completely defined, and specific labels are assigned to two disjointed sets connected by $S$ and $T$ by finding the minimum cost cut in the graph via graph cuts[13].

The graph cuts find the cut with the minimum cost among all the cuts, and the minimum cost cut problem can be solved by finding the maximum flow from $S$ to $T$ based on the theorem of Ford and Fulkerson[14]. The maximum flow(Fig. 1) saturates the set of edges in the graph, dividing the nodes into two disjointed parts corresponding to the minimum cut, and the value of the maximum flow is equal to the cost of the minimum cut[14]. Thus, since the maximum flow in a graph can assist with energy minimization for the labeling problem, the proposed method uses the maximum flow for global minimization of the energy function. Fig. 1 shows a pseudo code of the basic Ford-Fulkerson algorithm[14], where $f(u, v)$ and $\varepsilon(u, v)$ denote the flow and a weight between two vertices $u$ and $v$, respectively.

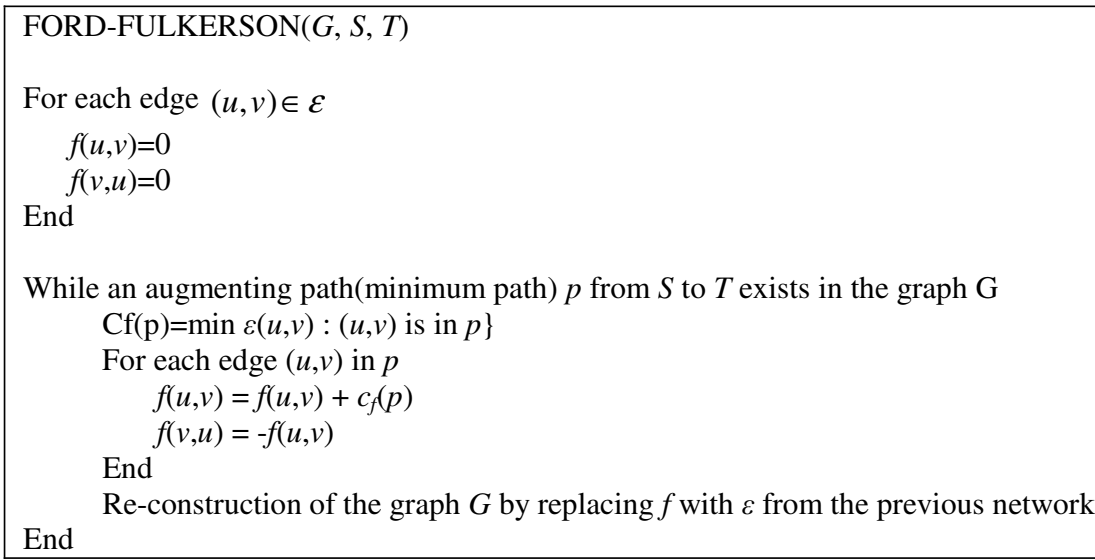

Fig. 1. Pseudo code of Basic Ford-Fulkerson Algorithm[13]

\subsection{Automatic Retrieval of Prior Data from Image}

Prior information for each label is required to assign suitable values into data terms of the energy function, and is manually obtained using interactive tools, e.g. foreground seeds and background ones in [4], in previous approaches.

To automatically obtain prior information for foreground segmentation, methods based on modeled background images were proposed[10,11]. Howe and Deschamps[10] used the difference $\delta_{p}$ between the background image and the input 
image as prior information for the background, and the data terms are defined as follows:

$$
D_{p}\left(f_{p}\right)=\left\{\begin{array}{cl}
\delta_{p} & \text { if } f_{p}={ }^{\prime} b g^{\prime} \\
2 \tau-\delta_{p} & \text { if } f_{p}={ }^{\prime} f g^{\prime}
\end{array}\right.
$$

This term was very sensitive to noises, as it used just the difference as prior information of the background, and made many holes within segmented foreground owing to inaccurate prior information of the foreground, which is assigned by given parameters $\tau$. Moreover, smooth terms can not express discontinuities between neighboring pixels, as they used Pott model, which is assigned by just given parameter $\tau$ and $\alpha($ Eq. 3).

$$
V_{p, q}\left(f_{p}, f_{q}\right)=\left\{\begin{array}{cl}
\tau \alpha & \text { if } f_{p} \neq f_{q} \\
0 & \text { if } f_{p}=f_{q}
\end{array}\right.
$$

Sun et al.[11] used GMM for prior information of the background, which is robust to the noises, but used constant values as prior information of the foreground based on an assumption that is uniform distribution in appearance of foreground objects, which restricts applicability of the proposed method. The data term of the energy function proposed by [11] is defined as follows:

$$
D_{p}\left(f_{p}\right)=\left\{\begin{array}{cl}
\arg \min _{k}\left\|\left(I_{p}-\boldsymbol{\mu}_{k}\right) \sum_{k}^{-\frac{1}{2}}\right\| & \text { if } f_{p}={ }^{\prime} b g^{\prime} \\
\text { const } & \text { if } f_{p}={ }^{\prime} f g^{\prime}
\end{array}\right.
$$

where $k$ is the number of Gaussian models, $\boldsymbol{\mu}_{\mathrm{k}}$ and $\boldsymbol{\Sigma}_{\mathrm{k}}$ are the mean and covariance matrix of $k^{\text {th }}$ Gaussian models, and $I_{p}$ is information of pixel $p$. The smooth term used the contrast term[14](Eq. 5) that encourages spatial coherence by penalizing discontinuities between neighboring elements $p$ and $q$ when adjacent elements are assigned different labels, allowing the capture of only gradient information between the labels.

$$
V_{p, q} \cdot\left|f_{p}-f_{q}\right|=\left\{\begin{array}{cl}
1-\frac{\tan ^{-1}\left(\left|\delta_{p q}\right|\right)}{\pi / 2} & \text { if } f_{p} \neq f_{q} \\
0 & \text { if } f_{p}=f_{q}
\end{array}\right.
$$

where $\delta_{p q}$ is difference between neighboring pixel $p$ and $q$.

The main problem of above mentioned methods is the accurate prior information for the foreground can not be obtained. Accordingly, this paper approximately segments foreground objects to efficiently estimate data terms for foreground segmentation. The foreground objects are segmented from each background image using background subtraction, and noises and shadows should be removed from the segmented objects to reduce errors of prior information for foreground objects. Noises can be removed by simple morphological operations. Removing the noises may cause to lose legitimate detail in the foreground silhouette, but the smooth term that assigns low costs if neighboring pixels are not similar, i.e. edges in images, and can 
compensate this problem, which means filling out the lost silhouette. Shadows in segmented foregrounds are removed based on an assumption that they have similar hue but lower brightness compared with background pixels, and a following equation shows the assumption.

$$
\text { Shadow }_{x y}= \begin{cases}1 & \text { if }\left|H u e_{x y}^{f g}-H u e_{x y}^{b g}\right|<\tau_{H} \text { and } B r t_{x y}^{f g}-B r t_{x y}^{b g}<0 \\ 0 & \text { otherwise }\end{cases}
$$

where $\mathrm{Hue}_{p}$ is a hue value in a pixel $p$ of input images, and $B r t_{x y}^{b g}$ is a brightness value in a pixel $p$ of background images. Fig. 2 shows an example of approximate foreground results. Fig. 2(b) shows a noiseless result of background subtraction by morphological operations, Fig. 2(c) shows an image that the gray color indicates shadow pixels, while Fig. 2(d) shows a result of an approximate foreground result.

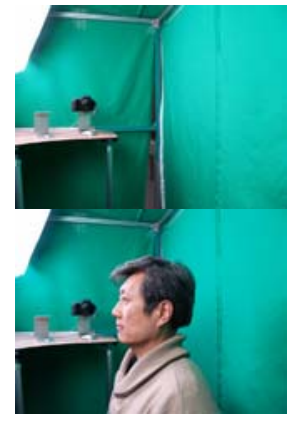

(a)

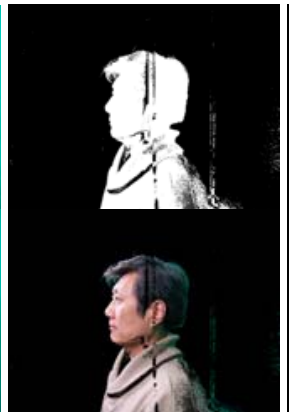

(b)

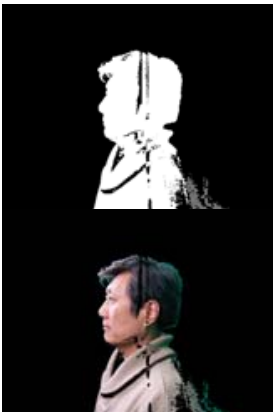

(c)

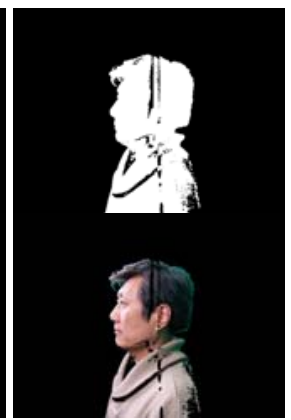

(d)

Fig. 2. Approximate foreground result: (a) input image and background image, (b) noiseless image, (c) shadow pixels indicated by graph color, and (d) result image

To obtain prior information for the foreground object, we use GMM that is robust to the noises, and data terms of the proposed method are defined as follows:

$$
D_{p}\left(f_{p}\right)= \begin{cases}\arg \min _{k}\left(\alpha_{k}^{b g} \cdot \frac{1}{2 \pi\left|\sum_{k}^{b g}\right|} \exp ^{-\frac{1}{2}\left(I_{p}-\mathbf{u}_{k}^{b g}\right)^{T} \sum_{k}^{b g}\left(I_{p}-\mathbf{u}_{k}^{b g}\right)}\right) & \text { if } f_{p}={ }^{\prime} b g^{\prime} \\ \arg \min _{k}\left(\alpha_{k}^{f g} \cdot \frac{1}{2 \pi\left|\sum_{k}^{f g}\right|} \exp ^{-\frac{1}{2}\left(I_{p}-\mathbf{u}_{k}^{f g}\right)^{T} \sum_{k}^{f g}\left(I_{p}-\mathbf{u}_{k}^{f g}\right)}\right) & \text { if } f_{p}={ }^{\prime} f g^{\prime}\end{cases}
$$

where $\mu_{k}^{f g}$ and $\sum_{k}^{f g}$ are the mean and covariance matrix of $k^{\text {th }}$ Gaussian models for the foreground. To completely construct the graph, these costs of data terms and smooth terms are set for the $t$-links and $n$-links in the graph, where $D_{p}\left(f_{p}={ }^{\prime} f g '\right)$ is set for $\{p, T\}$ and $D_{p}\left(f_{p}=^{\prime} f g^{\prime}\right)$ is set for $\{p, T\}$. 


\section{Experimental Results}

The computer used in the experiments consisted of intel Core2Quad Q6600 CPU and NVIDIA GeForce 8800 GTX graphic card, and we used Olympus E-500 cameras to obtain high resolution images. The software to develop the proposed system is implemented using Microsoft Visual C++ 6.0 and Olympus camera SDK, and the size of the input image is $800 \times 600$.

The experiment results are divided into two sub-subjects: 1) experimental results for foreground segmentation, and 2) 3D face reconstruction results.

\subsection{Experimental Results for Foreground Segmentation}

Three methods, background subtraction, Howe's graph cuts-based method[10], and the proposed method, was compared. The connected component error(CCE)(Eq. 8) criterion proposed in [10] was adopted for evaluation of each method, which takes only connected component of the segmented foreground that overlaps with ground truth into account, and Table 1 shows comparison of each method using CCE.

$$
C C E=\frac{\# \text { in false positives }+\# \text { in false negatives }}{\# \text { in ground truth }}
$$

Table 1. Comparison of foreground segmentation results

\begin{tabular}{c|c|c}
\hline Background Subtraction & Howe's & Proposed Method \\
\hline 0.287 & 0.161 & $\mathbf{0 . 1 1 5}$ \\
\hline
\end{tabular}
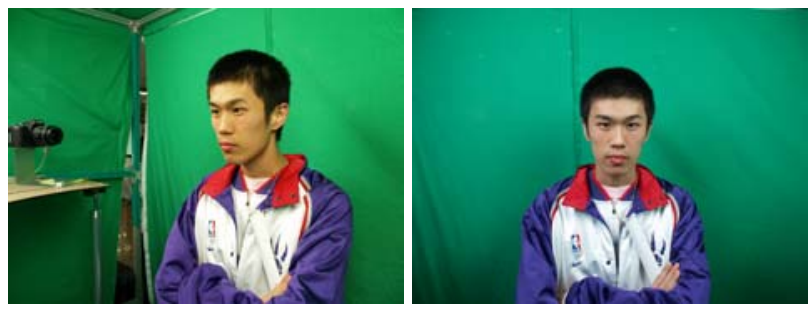

(a)
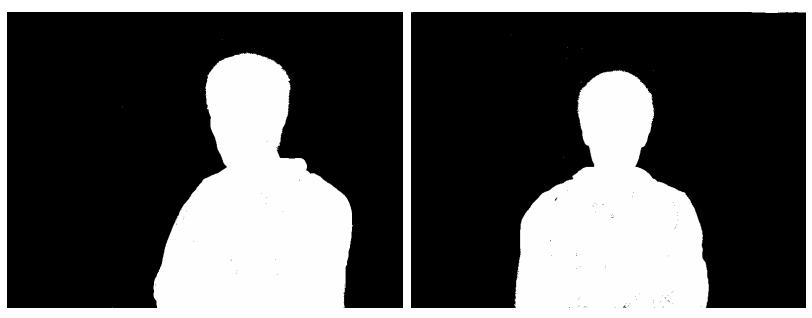

(b)
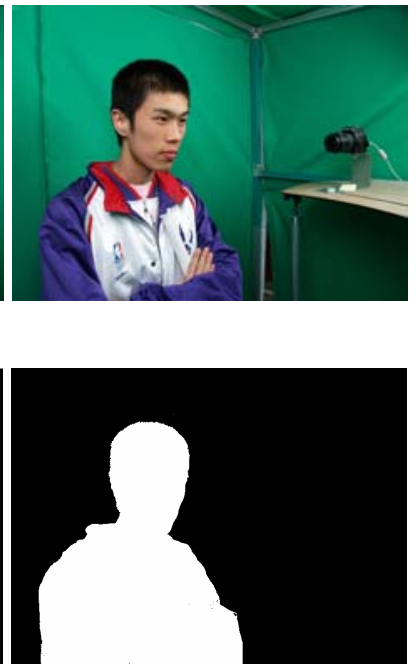

Fig. 3. Result images of proposed method: (a,c,e) input images, (b) result images 

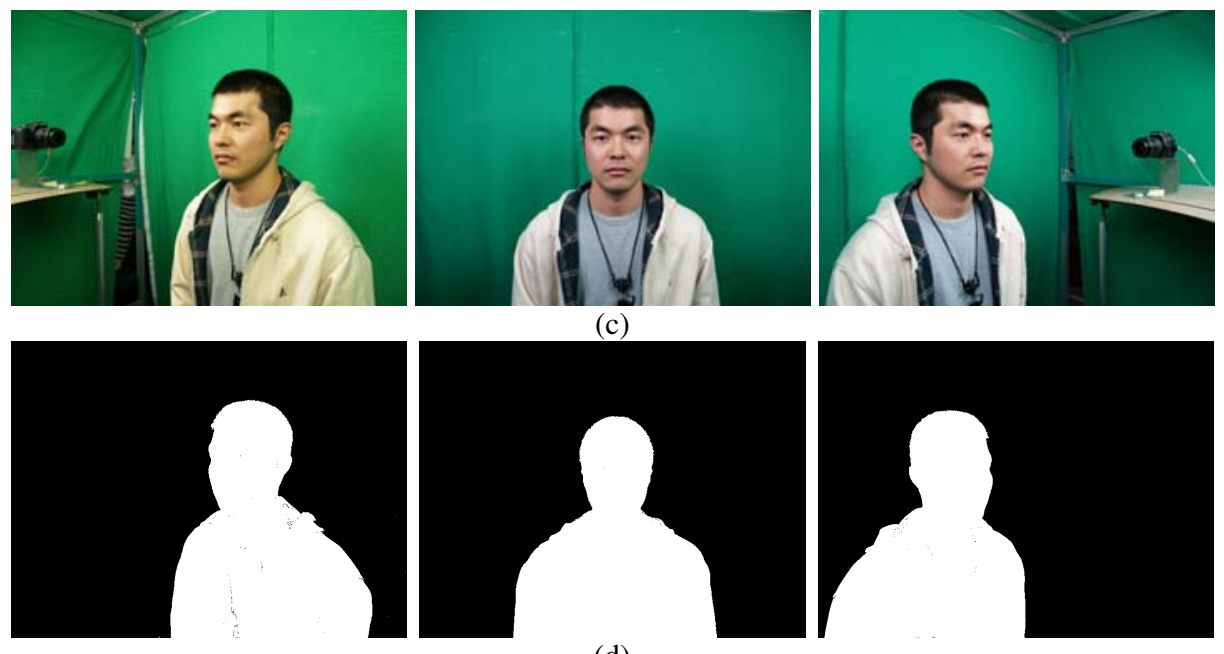

(d)
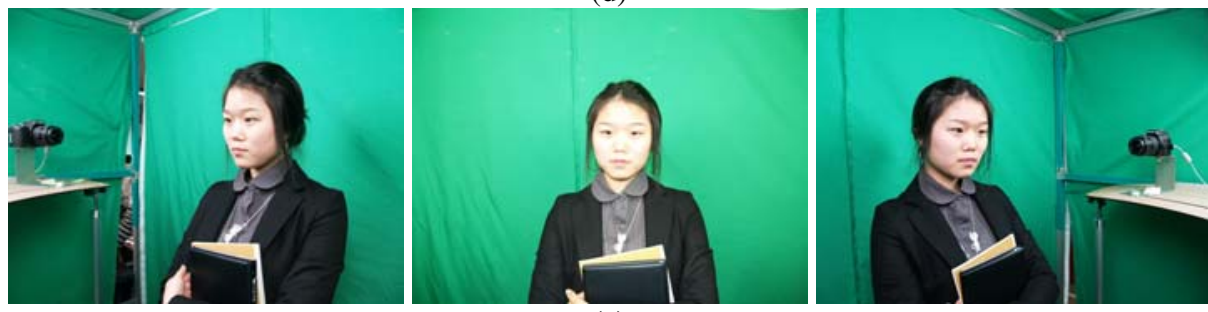

(e)
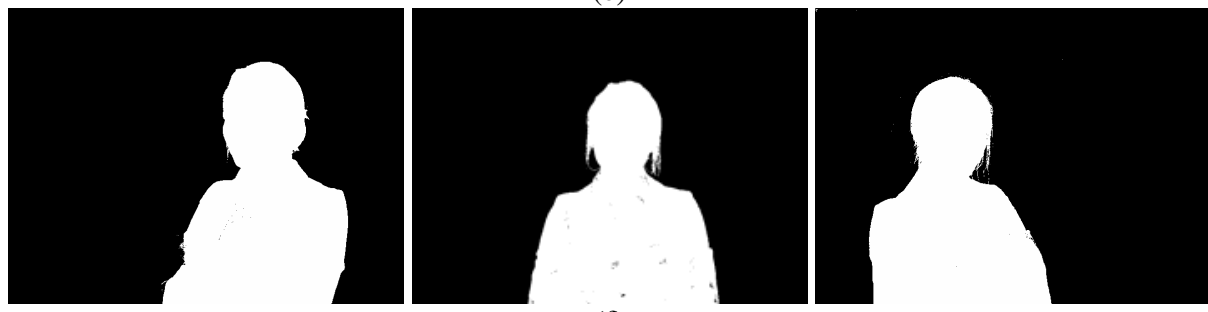

(f)

Fig. 3. (continued)

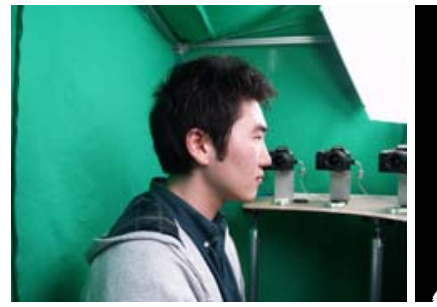

(a)

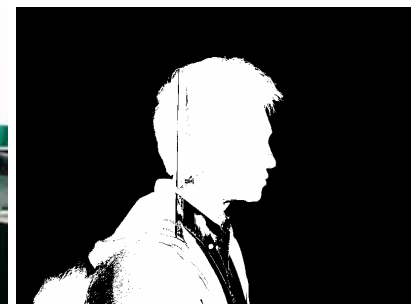

(b)

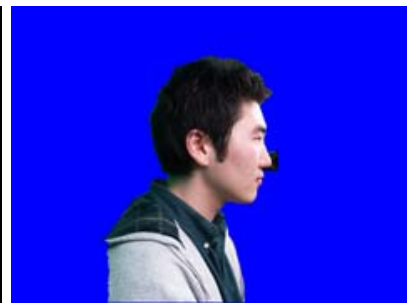

(c)

Fig. 4. Misclassification result: (a) input images, (b) background subtraction result, (c) output 
Fig. 3 shows the results of the proposed method, Figs. 3(a,c,e) shows input image, while Figs. 3(b,d,f) shows results images. Fig. 4 shows results of misclassification. This error was occurred when shadow on foreground was removed for foreground segmentation, and almost all misclassification of the proposed method was occurred on the side face in the experiments.

\subsection{D Face Reconstruction}

We reconstruct 3D objects based on segmented foreground objects, and 3D object reconstruction consists of two steps: Volume Intersection[1] and Object Carving[9](Fig. 5).

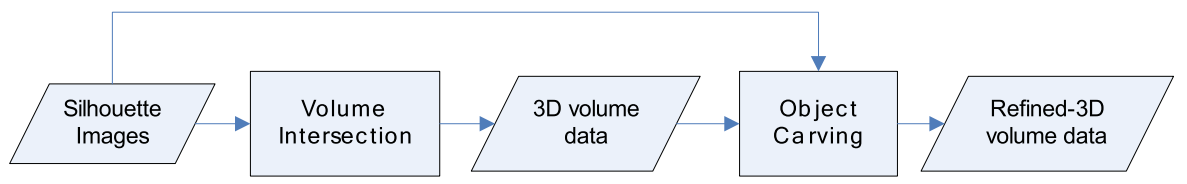

Fig. 5. Flow-chart of 3D object reconstruction

The Volume Intersection is performed using Silhouette Volume Intersection(SVI) algorithm[1] that is the most popular method to reconstruct 3D object from multiview images. This method first projects silhouette images transformed using a calibration matrix onto each plane of the voxel space from the corresponding camera viewpoint, and then calculates the intersection among transformed silhouette images.

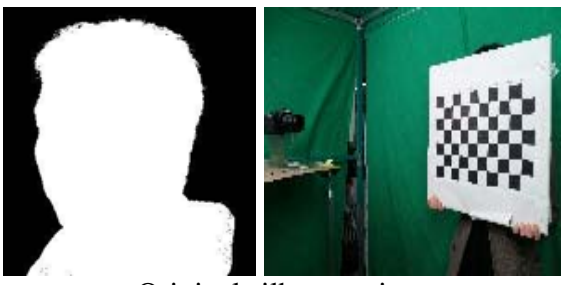

Original silhouette image

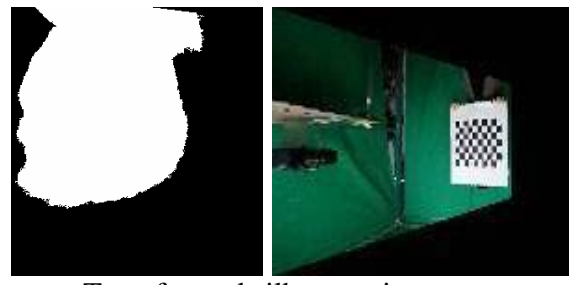

Transformed silhouette image

(a)
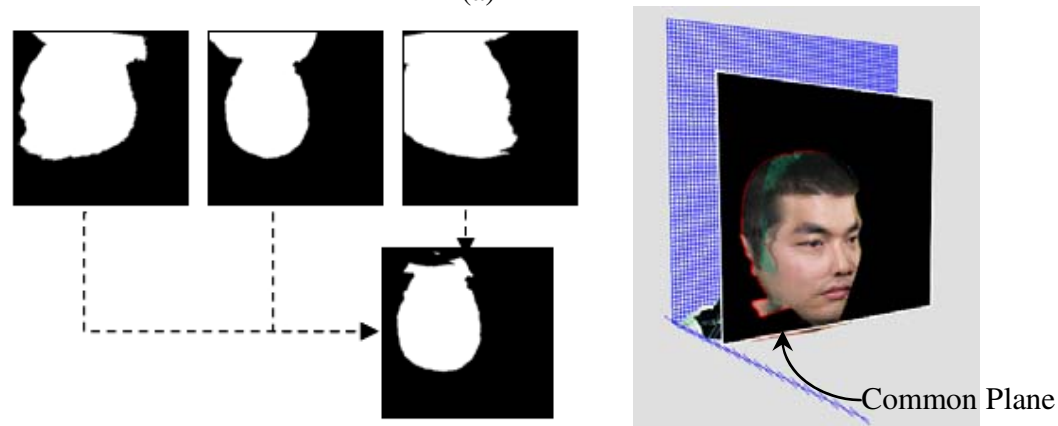

(b)

Fig. 6. Sub-steps of SVI: (a) plane image generation step, (b) plane image intersection step 


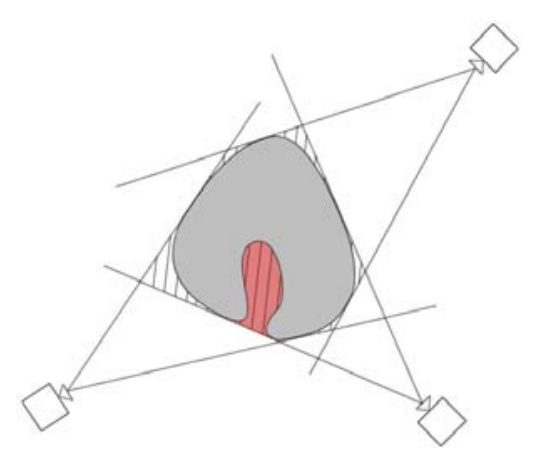

Fig. 7. Problem of SVI

As shown in Fig. 6, the SVI algorithm consists of two sub-steps: plane image generation and plane image intersection. In the plane image generation, all the silhouette images captured by multi-camera are projected onto a common plane using calibration matrix of each camera(Fig. 6(a)). Then, we compute 2D intersection of all projected silhouette images on each plane(Fig. 6(b)). Here, the common plane is perpendicular to the z-axis, and is moved from the common base plane (gray-colored check plane in the right image of Fig. 6(b)) until no silhouette images are projected onto the common plane. The results on each plane are called visual hull, and Fig. 6(b) shows the visual hull and the common plane of the foreground object. However, almost all image-based 3D reconstruction methods can make only the convex visual hull(Fig. 7), e.g. darker gray regions can not be expressed in reconstructed objects in Fig. 7.

Object Carving is used to solve this problem. Generally, the photo-consistency between neighboring silhouette images is used to reconstruct more accurate shapes. As shown in Fig. 8, we find a point $p$ on a silhouette image A and a point $q$ on a silhouette image $B$ that two points correspond to the $3 \mathrm{D}$ point $\mathrm{P}$ on the visual hull, and compute the epipolar line $l$ on silhouette image B that the line passes the point $q$. If $p$ and $q$ do not have photo-consistency, we delete the point $\mathrm{P}$ and compare $p$ with $q$ ' that

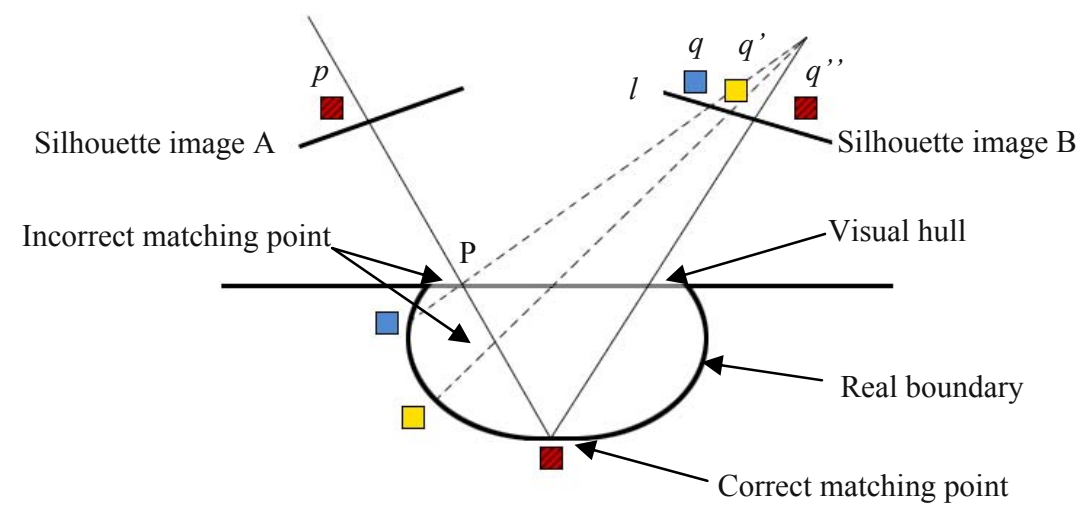

Fig. 8. Object Carving 


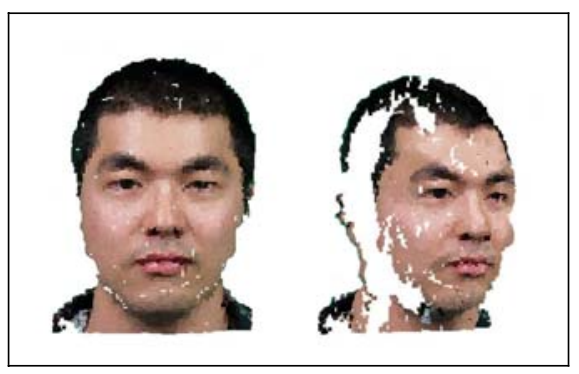

Fig. 9. Result of 3D face reconstruction with texture mapping
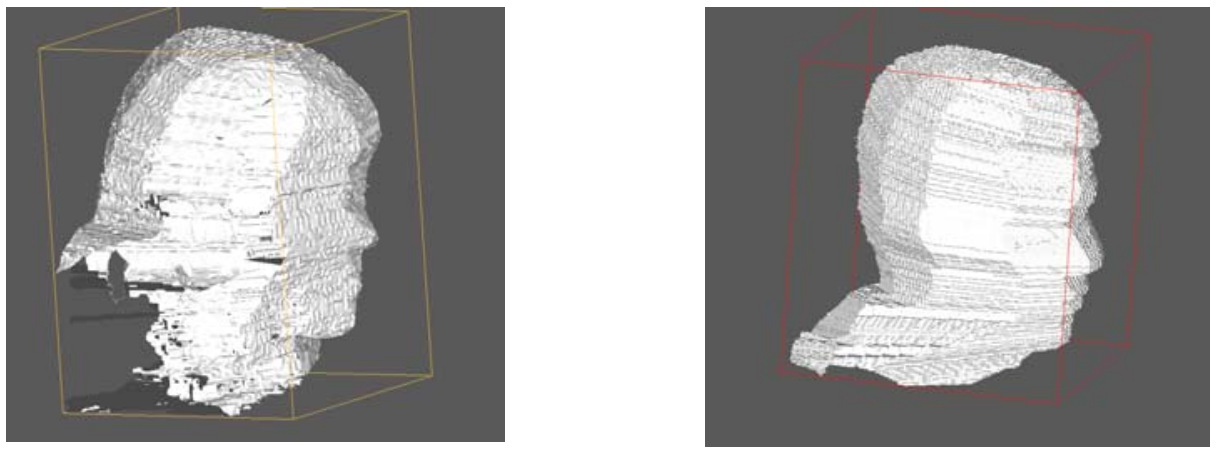

Fig. 10. Results 3D face reconstruction without texture mapping: (a) using background subtraction, (b) using proposed method

is a neighbor of the $q$ until two points have the photo-consistency. Fig. 8 shows an example of results of $3 \mathrm{D}$ face reconstruction, and we paint colors to 3D faces using texture mapping.

Fig. 9 shows results of $3 \mathrm{D}$ face reconstruction without texture mapping. Since almost all misclassification of the proposed method was occurred on the side face in the experiments. Therefore, we did not use the side face to construct $3 \mathrm{D}$ face, and thus the side faces did not have textures. Fig. 10(a) shows a result using background subtraction, while Fig. 10(b) shows a result using the proposed method. As shown in Fig. 10, Volume Intersection and Object Carving to reconstruct 3D objects must have accurate foreground objects segmented from background images.

\section{Conclusion}

In this paper, we proposed better foreground segmentation method for 3D face reconstruction using graph cuts. Stereo vision techniques were generally used for 3D reconstruction, and depth information was used as prior information for data terms of energy functions. However, it was difficult to calculate depth information in $3 \mathrm{D}$ face reconstruction, as the most of faces had homogeneous regions. Therefore, we used Volume Intersection and Object Carving that does not use depth information for 3D face reconstruction, which should have accurate foreground objects. 
In this paper, foreground objects from each input image were approximately segmented from each background image using background subtraction to estimate data terms of energy functions, and noises and shadows were removed from the segmented objects to reduce errors of prior information for foreground objects. Removing the noises and shadows cased to lose detail in the foreground silhouette, but smooth terms of energy functions filled out the lost silhouette. As a result, the proposed method can segment more precise foreground objects by globally minimizing the energy function composed of smooth terms and approximately estimated data terms using graph cuts.

However, if shadows were located in foreground objects, the regions were also removed at the final foreground objects. Therefore, we will investigate how to remove more accurate shadow regions.

Acknowledgement. This work was supported by the Soongsil University Research Fund.

\section{References}

1. Laurentini, A.: The Visual Hull Concept for Silhouette-based Image Understanding. IEEE Transactions on Pattern Analysis and Machine Intelligence 16(2), 150-162 (1994)

2. Wayne Power, P., Schoonees, J.: Understanding Background Mixture Models for Foreground Segmentation. In: Proceedings of Image and Vision Computing, New Zealand, pp. 267-271 (2002)

3. Image Processing Toolbox, ch. 9: Morphological Operations, The Mathworks (2001)

4. Li, Y., Sun, J., Tang, C-K., Shum, H-Y.: Lazy Snapping. ACM Transactions on Graphics 23(3), 303-308 (2004)

5. Boykov, Y., Funka-Lea, G.: Graph Cuts and Efficient N-D Image Segmentation. International Journal of Computer Vision 70(2), 109-131 (2006)

6. Rother, C., Kolmogorov, V., Blake, A.: GrabCut - Interactive Foreground Extraction using Iterated Graph Cuts. ACM Transactions on Graphics 23(3), 309-314 (2004)

7. Ahn, J-H., Kim, K., Byun, H.: Robust Object Segmentation using Graph Cut with Object and Background Seed Estimation. In: Proceedings of International Conference on Pattern Recognition, vol. 2, pp. 361-364 (2006)

8. Kolmorogov, V., Criminisi, A., Blake, A., Cross, G., Rother, C.: Probabilistic Fusion of Stereo with Color and Contrast for Bilayer Segmentation. IEEE Transactions on Pattern Analysis and Machine Intelligence 28(9), 1480-1492 (2006)

9. Kutulakos, K.N., Seitz, S.M.: A Theory of Shape by Space Carving. International Journal of Computer Vision 38(3), 199-218 (2000)

10. Howe, N.R., Deschamps, A.: Better Foreground Segmentation through Graph Cuts. Technical Report (2004), http://arxiv.org/abs/cs.CV/0401017

11. Sun, Y., Li, B., Yuan, B., Miao, Z., Wan, C.: Better Foreground Segmentation for Static Cameras via New Energy Form and Dynamic Graph-cut. In: Proceedings of International Conference on Pattern Recognition, vol. 2, pp. 49-52 (2006)

12. Boykov, Y., Veksler, O., Zabih, R.: Fast Approximate Energy Minimization via Graph Cuts. IEEE Transactions on Pattern Analysis and Machine Intelligence 23(11), 1222-1239 (2001)

13. Ford, L., Fulkerson, D.: Flows in Networks. Princeton University Press, Princeton (1962)

14. Kumar, M.P., Torr, P.H.S, Zisserman, A.: Obj Cut. Proceedings of Computer Vision and Pattern Recognition 1, 18-25 (2005) 